\title{
Effect of FK506 in reducing scar formation by inducing fibroblast apoptosis after sciatic nerve injury in rats
}

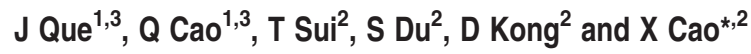

We previously demonstrated that FK506, a generally applied immunosuppressant in organ transplantation, could promote peripheral nerve regeneration through reducing scar formation. However, little is known about how FK506 reduces scar formation. Herein we investigated the influence of FK506 on fibroblast proliferation and its correlation with scar formation after sciatic nerve injury in rats, and further explored the effect of FK506 on fibroblast proliferation and apoptosis in vitro. Masson staining and immunohistochemistry revealed that scar area and fibroblast number in the nerve anastomosis of sciatic nerveinjured rats were significantly reduced after FK506 administration. The scar area had a significant positive correlation with the fibroblast number, as detected by linear correlation analysis. CCK-8 assay and flow cytometry indicated that FK506 also inhibited proliferation and induced apoptosis of fibroblasts in vitro. It was primarily phosphorylation of JNK and ERK that were activated during the apoptosis of fibroblast. Pretreatment of cells with JNK inhibitor, SP600125, or ERK inhibitor, PD98059, could inhibit FK506-induced fibroblast apoptosis, respectively. Moreover, simultaneous application of both inhibitors had additive roles in cell protection from apoptosis. These results suggest that FK506-induced fibroblast apoptosis contributes to the suppression of fibroblast proliferation and then results in the reduction of scar formation in sciatic nerve-injured rat, and that JNK and ERK are involved in FK506-induced fibroblast apoptosis.

Cell Death and Disease (2013) 4, e526; doi:10.1038/cddis.2013.56; published online 7 March 2013

Subject Category: Experimental medicine

Following traumatic peripheral nerve injury, proliferation of scar tissue from the epineurium will result in impediment to the regenerating axons that need to traverse the injury site. ${ }^{1-5} \mathrm{~A}$ reduction in scar formation at a site of nerve repair is associated with better recovery of neural function. ${ }^{6,7}$ It has been demonstrated that FK506, a generally applied immunosuppressant in organ transplantation, has powerful effect of promoting axon regeneration ${ }^{8-12}$ through its immunosuppressive action ${ }^{13}$ and neurotrophic action. ${ }^{14-18}$ Moreover, our previous study ${ }^{19}$ found that FK506 could promote peripheral nerve regeneration and accelerate the recovery of neural function by reducing scar formation. It is believed that increased collagen biosynthesis from the proliferative fibroblasts contributes to scar formation at the repair site, and preventing fibroblast proliferation can reduce scar area. ${ }^{20,21}$ In order to gain further insight into the mechanism of action of FK506, this study investigated the effect of FK506 on fibroblast proliferation and its correlation with scar formation in sciatic nerve-injured rat model, and further explored the influence of FK506 on fibroblast proliferation and apoptosis in vitro and its possible cell signaling. The results from this study may provide further explanation for the effect of FK506 on promoting peripheral nerve regeneration.

\section{Results}

Effect of FK506 on scar formation and fibroblast proliferation in histological analysis. At 4 weeks after establishing the nerve injury model, Masson staining showed extensive collagen tissue hyperplasia in nerve anastomosis of the model group (Figure 1A), as seen by the blue staining. Immunohistochemistry showed that a large amount of fibroblasts mixed with fibrocytes appeared in the model group (Figure 1a). In the FK506 group (Figures 1B and b), collagen tissue hyperplasia and fibroblasts mixed with fibrocytes obviously decreased in nerve anastomosis compared with those of the model group. Meanwhile, collagen tissue and fibroblasts were rarely visible in nerve anastomosis of the normal control group (Figures $1 \mathrm{C}$ and $\mathrm{C}$ ).

Effect of FK506 on fibroblast number in sciatic nerve anastomosis of rats and its correlation with scar area. As shown in Figure 2a, the scar area of nerve anastomosis was significantly increased in the model group $\left(4.36 \pm 0.68 \mathrm{~mm}^{2}\right)$ compared with the normal control group $\left(0.37 \pm 0.14 \mathrm{~mm}^{2}, P<0.01\right)$, and significantly decreased in the FK506 group $\left(1.61 \pm 0.42 \mathrm{~mm}^{2}\right)$ compared with the model group $(P<0.05)$. As shown in Figure $2 \mathrm{~b}$, the fibroblast

\footnotetext{
${ }^{1}$ Department of Intensive Care Unit, The First Affiliated Hospital of Nanjing Medical University, Nanjing, China and ${ }^{2}$ Department of Orthopedics, The First Affiliated Hospital of Nanjing Medical University, Nanjing, China

*Corresponding author: X Cao, Department of Orthopedics, The First Affiliated Hospital of Nanjing Medical University, 300 Guangzhou Road, Nanjing 210029 , China. Tel: + 86013002505801 ; Fax: + 8625 83724440; E-mail: xiaojiancao@ gmail.com

${ }^{3}$ These authors equally contributed to this work.

Keywords: scar formation; FK506; apoptosis; fibroblast; cell signaling

Abbreviations: CCK-8, Cell Counting Kit-8; DMSO, dimethyl sulfoxide; PBS, phosphate buffered saline; MAPK, mitogen-activated protein kinase; Apaf-1, apoptotic protease-activating factor- 1 .

Received 08.8.12; revised 30.1.13; accepted 04.2.13; Edited by E Candi
} 


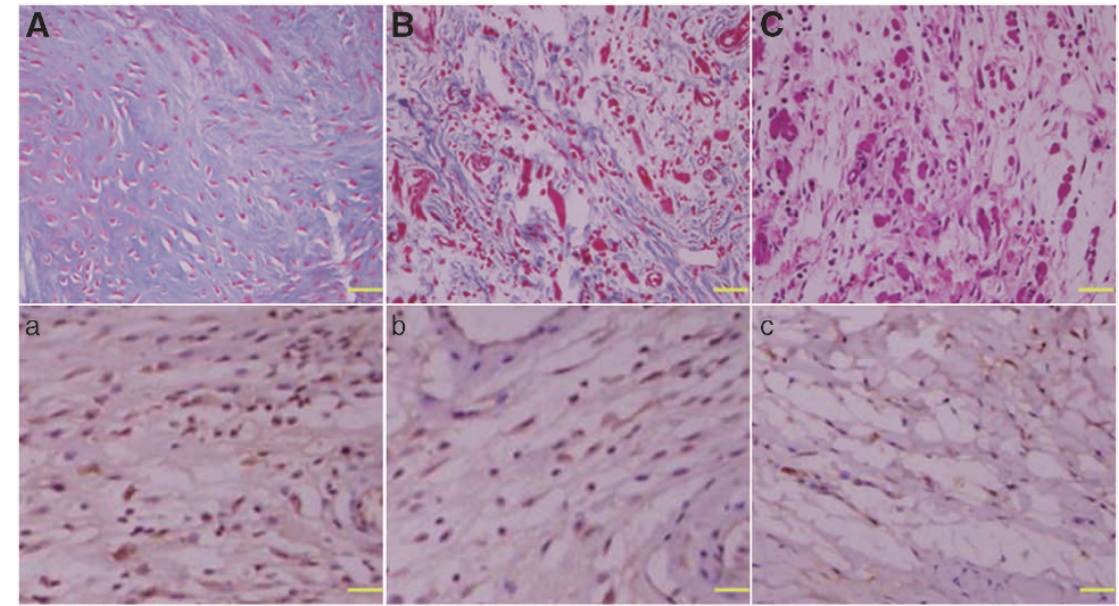

Figure 1 Representative histological images of collagen tissue and fibroblast proliferation in sciatic nerve anastomosis of rats. The sections obtained from model group ( $\mathbf{A}$ and a), FK506 group (B and b) and normal control group ( $\mathbf{C}, c)$ were stained with Masson's trichrome $(\mathbf{A}, \mathbf{B}$ and $\mathbf{C})$ and anti-TGF- $\beta(a, b$ and $c)$, respectively. The collagen tissues and fibroblasts appear blue in the sections stained with Masson's trichrome or anti-TGF- $\beta$. The density of collagen tissue (B), and the number of fibroblast (b) in the sections of FK506 group were significantly less than those (A, a) of model group. Images $\times 400$ magnified, scale bar $20 \mu \mathrm{m}$
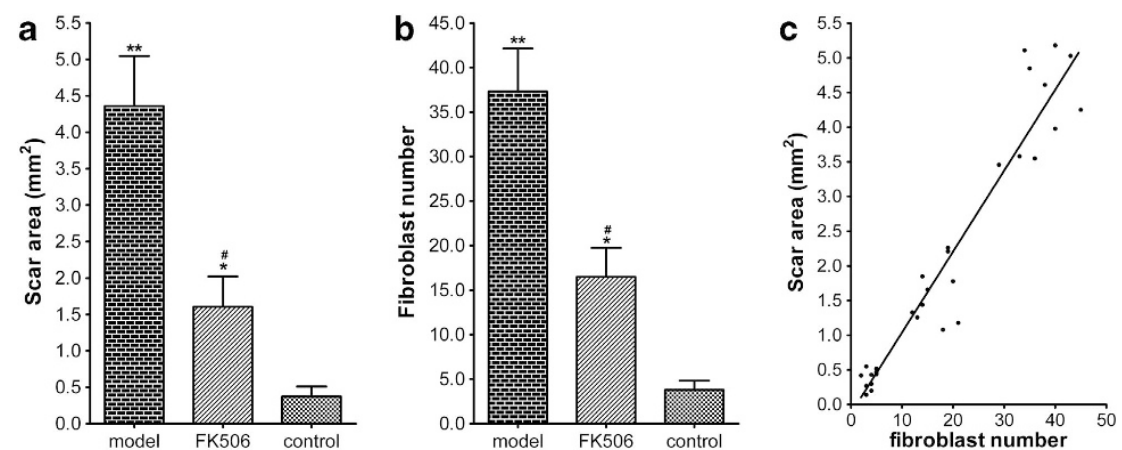

Figure 2 Effect of FK506 on fibroblast counts and scar area in sciatic nerve anastomosis of rats. (a) The area of scar tissue for each rat was defined as the mean scar area of six sections and the data were expressed as mean \pm S.D. in $\mathrm{mm}^{2} .{ }^{\star} P<0.05$, ${ }^{\star} P<0.01$ compared with the area of scar tissue in normal control group. ${ }^{\#} P<0.05$, compared with that in model group. (b) Fibroblast counting was expressed as the number per counting area. ${ }^{*} P<0.05,{ }^{* *} P<0.01$ compared with the number of fibroblast in normal control group. ${ }^{\#} P<0.05$, compared with that in model group. (c) Correlation between scar area and fibroblast number (linear correlation analysis). The scar area ( $\mathrm{mm}^{2}$ ) was apparently positively correlated with the fibroblast number (number per counting area; $r=0.9915, P<0.0001$ )

number of nerve anastomosis was significantly increased in the model group $(37.3 \pm 4.85)$ compared with the normal control group $(3.8 \pm 1.03, \quad P<0.01)$, and significantly decreased in the FK506 group (16.5 \pm 3.24$)$ compared with the model group $(P<0.05)$. Linear correlation analysis results showed that the scar area of nerve anastomosis augmented with increased fibroblast number. Thus, the scar area had a significant positive correlation with the fibroblast number $(r=0.9915, P<0.0001)$, suggesting that FK506 reduces scar formation by inhibiting fibroblast proliferation (Figure 2c).

FK506 inhibited fibroblast proliferation in vitro in a dose-dependent manner. Rat skin fibroblasts were treated with FK506 at increasing concentrations for $8 \mathrm{~h}$. The Cell Counting Kit-8 (CCK-8) assay demonstrated that FK506 could induce a dramatic loss in the viability of fibroblasts. Cell viability reached a relatively minimal level at $75 \mu \mathrm{M}$ (Figure 3). FK506 is an effective inhibitor of fibroblast proliferation in vitro and this inhibitory effect is dose-dependent.
FK506 induced significant apoptosis of fibroblast. As the CCK-8 assay demonstrated that FK506 could inhibit the proliferation of fibroblast in a dose-dependent manner, flow cytometry was further used to identify the types of cell death. As shown in Figure 4, fibroblasts showed a dosedependent apoptosis, including early as well as late apoptotic cell death. The mean percentage of apoptotic cell death was about $(14.7 \pm 6.89),(45.7 \pm 7.05)$ and (86.8 \pm $6.83) \%$ in cells treated with $12.5,25$ and $50 \mu \mathrm{M}$ FK506, respectively. In contrast, the cells that had not been treated with FK506 demonstrated normal cell viability without abnormal cell death.

Morphological changes by fluorescence microscopy. In order to characterize the pattern of apoptotic status mediated by FK506 in fibroblasts, morphological examinations were performed. As shown in Figure 5, normal fibroblasts exhibited intact nuclei and adqulis chromatin. In comparison, after FK506 treatment and fluorescence microscopy using Hoechst 33342 staining, shrunken cells with condensed or fragmented nuclei were observed. In cells treated with $50 \mu \mathrm{M}$ 


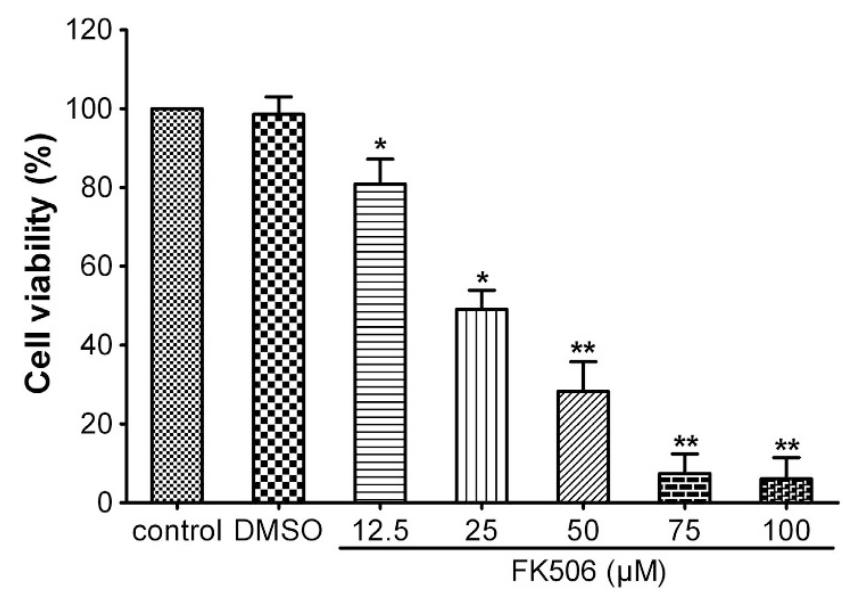

Figure 3 Effect of FK506 on fibroblast proliferation in vitro. Rat skin fibroblasts were treated with increasing concentrations of FK506 for $8 \mathrm{~h}$. The viable cells were measured by CCK-8 assay. FK506 inhibited fibroblast proliferation in a dosedependent manner. Cell viability reached a relatively minimal level at $75 \mu \mathrm{M}$. ${ }^{\star} P<0.05,{ }^{* *} P<0.01$ compared with control. All experiments were preformed three times with comparable results
FK506, significant chromatin agglutination and nuclear fragmentation, both characteristic of apoptosis, were observed.

FK506 activated JNK and ERK, and enhanced expressions of cytosolic cytochrome $c$ and cleaved caspase-3. JNK, ERK, cytochrome $c$ and cleaved caspase3 are all known to be involved in the regulation of apoptosis. Their expression levels after FK506 treatment for $8 \mathrm{~h}$ were detected by western blotting. As shown in Figure 6, the levels of GAPDH expression were comparable among the negative control group, the dimethyl sulfoxide (DMSO) group and the three FK506 treatment groups. In contrast, phosphorylation of JNK ( $p-J N K)$ could not be activated in either the negative control group or the DMSO group. Increasing expression of p-JNK was observed in fibroblasts after FK506 treatment at increasing concentrations; this expression peaked at the concentration of $50 \mu \mathrm{M}$. This suggests that JNK can be activated by FK506. The kinetics and the extent of phosphorylation of ERK ( $p$-ERK), cytosolic cytochrome $c$ and cleaved caspase-3 had a similar pattern of increase
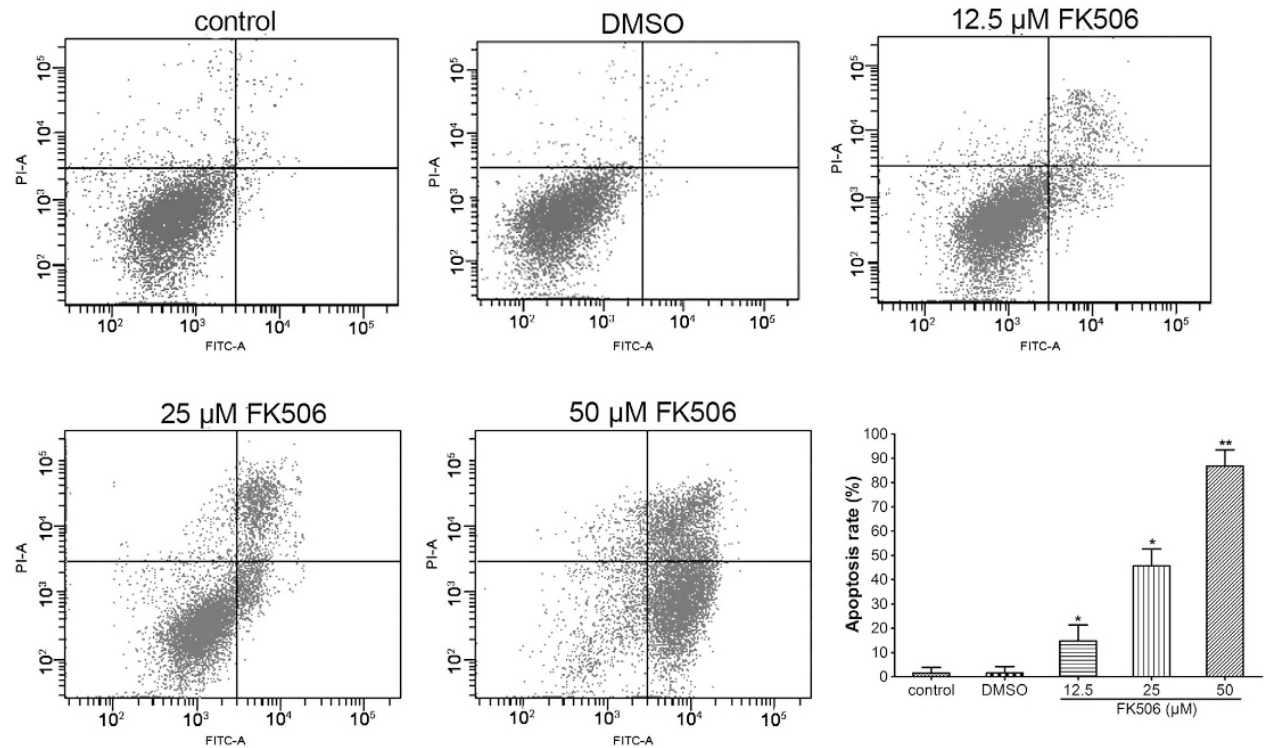

Figure 4 FK506 induced significant apoptosis of fibroblasts. Cells were incubated either in the absence of (control) or in the presence of FK506 (12.5, 25 and $50 \mu \mathrm{M})$ for $8 \mathrm{~h}$. Fibroblasts showed a dose-dependent apoptosis through the detection of flow cytometry. The mean percentage of apoptotic cell death was about (14.7 \pm 6.89 ), $(45.7 \pm 7.05)$ and $(86.8 \pm 6.83) \%$ in cells treated with $12.5,25$ and $50 \mu \mathrm{M}$ FK506, respectively. ${ }^{*} P<0.05,{ }^{* *} P<0.01$ compared with control

control

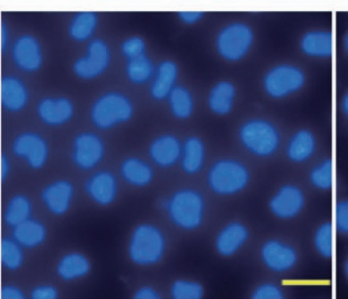

DMSO

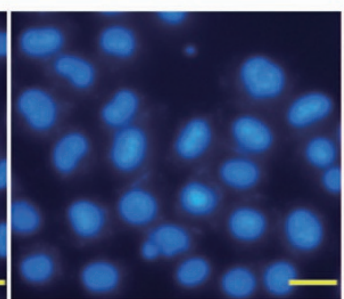

12.5 MM FK506

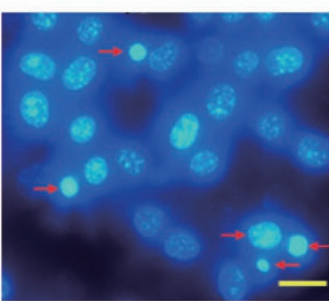

$25 \mu \mathrm{M}$ FK506

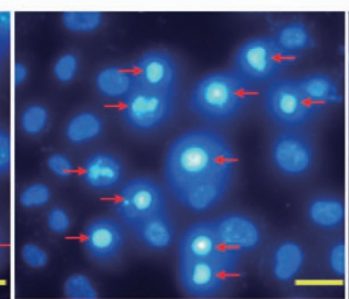

$50 \mu \mathrm{M}$ FK506

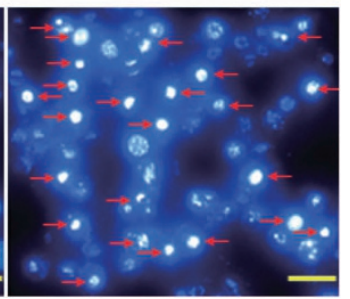

Figure 5 Morphological features of fibroblast apoptosis induced by FK506. Cells were treated with increasing concentrations of FK506 for $8 \mathrm{~h}$. Following Hoechst 33342 staining, the morphological features of apoptosis were observed by fluorescence microscopy. Normal fibroblasts exhibited intact nuclei and adqulis chromatin. Shrunken cells with condensed or fragmented nuclei were observed after FK506 treatment. Arrows showing condensed or fragmented nuclei. Images $\times 400$ magnified, scale bar $5.5 \mu \mathrm{m}$ 

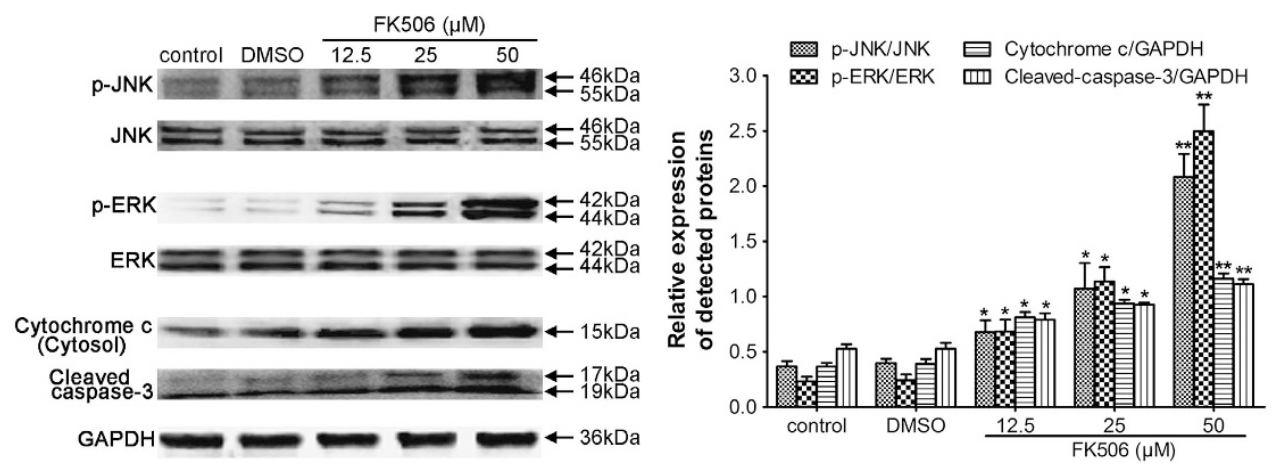

Figure 6 Effect of FK506 on protein expressions of p-JNK, p-ERK, cytosolic cytochrome $c$ and cleaved caspase-3. Cells were incubated either in the absence of (control) or in the presence of FK506 (12.5, 25 and $50 \mu \mathrm{M})$ for $8 \mathrm{~h}$, and protein expressions of p-JNK, p-ERK, cytosolic cytochrome $c$ and cleaved caspase-3 were determined using western blotting. Increasing expressions of $p$-JNK, p-ERK, cytosolic cytochrome $c$ and cleaved caspase-3 were observed in fibroblasts after FK506 treatment at increasing concentrations, and peaked at the concentration of $50 \mu \mathrm{M}$. ${ }^{*} P<0.05,{ }^{* *} P<0.01$ compared with control

resulting from FK506 treatment. The highest expressions of p-ERK, cytosolic cytochrome $c$ and cleaved-caspase-3 was reached at the FK506 concentration of $50 \mu \mathrm{M}$. FK506 also activated ERK, and enhanced expression of cytosolic cytochrome $c$ and cleaved caspase-3.

JNK inhibitor, SP600125, or/and ERK inhibitor, PD98059, reduced FK506-induced apoptosis of fibroblasts. The effects of JNK inhibitor, SP600125, and ERK inhibitor, PD98059, were investigated to demonstrate the role of JNK and ERK in FK506-induced apoptosis. As shown in Figure 7, preincubation with SP600125 or PD98059 for $30 \mathrm{~min}$ before $50 \mu \mathrm{M}$ FK506 treatment significantly reduced the apoptotic rate. The mean percentage of apoptotic cell death decreased to $(61.1 \pm 7.18)$ and $(67.6 \pm 7.59) \%$ in cells pretreated with SP600125 and PD98059, respectively, compared with that of cells treated with $50 \mu \mathrm{M}$ FK506 alone $(87.9 \pm 6.69) \%(P<0.05)$. In addition, preincubation with the two inhibitors together further decreased the apoptotic percentage to (39.87 \pm $4.57) \%$, compared with SP600125 or PD98059 pretreatment, respectively $(P<0.05)$.

JNK inhibitor, SP600125, or/and ERK inhibitor, PD98059, weakened expressions of $p$-JNK or p-ERK and cleaved caspase-3. As shown in Figure 8, SP600125 prevented both the phosphorylation of JNK and the expressions of cytosolic cytochrome $c$ and cleaved-caspase-3. However, PD98059 only prevented the phosphorylation of ERK and the expression of cleaved caspase-3, and had no effect on the expression of cytosolic cytochrome $c$. Moreover, simultaneous application of both inhibitors further decreased the expression of cleaved caspase-3 $(P<0.05)$, and almost had no influence on the expressions of p-JNK, p-ERK and cytosolic cytochrome $c$, compared with SP600125 or PD98059 pretreatment, respectively.

\section{Discussion}

Following traumatic peripheral nerve injury, fibroblasts were activated by inflammatory cytokines and growth factors, such as transforming growth factor- $\beta$ (TGF- $\beta$ ) and basic fibroblast growth factor. Proliferative fibroblasts produce a great amount of collagen fibers that contribute to scar formation at the nerve anastomotic sites, and preventing fibroblast proliferation can reduce scar area. ${ }^{20,21}$ In this study, multiple parameters including the histological analysis, the area of scar tissue and the number of fibroblasts were used to evaluate the effect of FK506 on fibroblast proliferation and scar formation in rats' model after sciatic nerve injury. Furthermore, we performed successive transversal sections through the anastomotic sites to make the scar area analysis and fibroblast counting in histological evaluation. Therefore, the mean values could reflect the amount of scar area and fibroblast number, and it was a valid method to the quantitative evaluation of fibroblast proliferation and scar formation.

With FK506 gavage ( $4 \mathrm{mg} / \mathrm{kg}$ per day) for 4 weeks after sciatic nerve injury, the scar area and fibroblast number were significantly decreased in the FK506 group compared with the model group. These results showed that FK506 could inhibit fibroblast proliferation and reduce scar formation at the nerve anastomotic sites. Moreover, correlation analysis indicated that the scar area was positively correlated with the fibroblast number, which further confirmed that FK506 can reduce scar formation in some way by inhibiting fibroblast proliferation after sciatic nerve injury in rats.

The study further investigated the influence of FK506 on fibroblast proliferation in vitro. The CCK-8 assay demonstrated that FK506 could inhibit the proliferation of cultured rat skin fibroblasts in a dose-dependent manner. Then the effect of FK506 on fibroblast apoptosis in vitro was examined. Hoechst 33342 staining showed that the fibroblasts subjected to FK506 exhibited condensed or fragmented nuclei. Furthermore, flow cytometric analysis showed a significant increase, in a dose-dependent manner, in the percentage of apoptotic cells. The more the apoptotic cells, the less the cells available for proliferation. Thus, these results suggest that FK506-induced fibroblast apoptosis contributes to the suppression of fibroblast proliferation and then results in the reduction of scar formation in sciatic nerve-injured rat.

Furthermore, we explored the cell signaling that may be involved in FK506-induced fibroblast apoptosis. We found that fibroblast apoptosis induced by FK506 could be inhibited by 

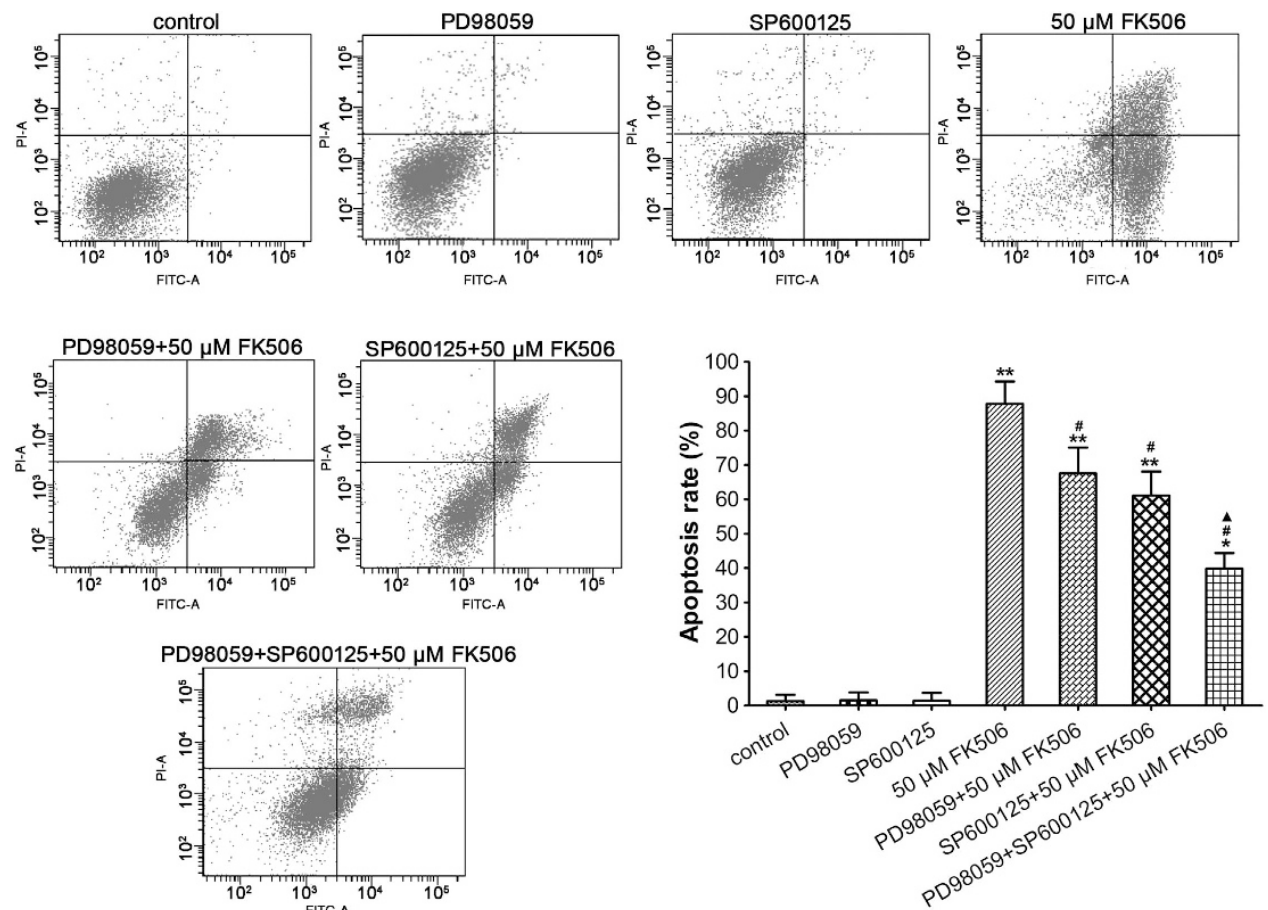

Figure 7 JNK inhibitor, SP600125, or/and ERK inhibitor, PD98059, reduced FK506-induced apoptosis of fibroblasts. Cells were preincubated with JNK inhibitor, SP600125 $(40 \mu \mathrm{M})$, or/and ERK inhibitor, PD98059 $(60 \mu \mathrm{M})$, for $30 \mathrm{~min}$ before treatment with $50 \mu \mathrm{M}$ FK506 for $8 \mathrm{~h}$. The apoptosis rate was detected by flow cytometry. The mean percentage of apoptotic cell death decreased to $(61.1 \pm 7.18)$ and $(67.6 \pm 7.59) \%$ in cells pretreated with SP600125 and PD98059, respectively, compared with that of cells treated with $50 \mu \mathrm{M}$ FK506 alone $(87.9 \pm 6.69) \%$. Preincubation with the two inhibitors together further decreased the apoptotic percentage to $(39.87 \pm 4.57) \%$, compared with SP600125 or PD98059 pretreatment, respectively. ${ }^{*} P<0.05$, ${ }^{\star}{ }^{\star} P<0.01$, compared with control. ${ }^{\#} P<0.05$ compared with cells treated with $50 \mu \mathrm{M}$ FK506 alone. $\Delta P<0.05$ compared with SP600125 or PD98059 pretreatment, respectively
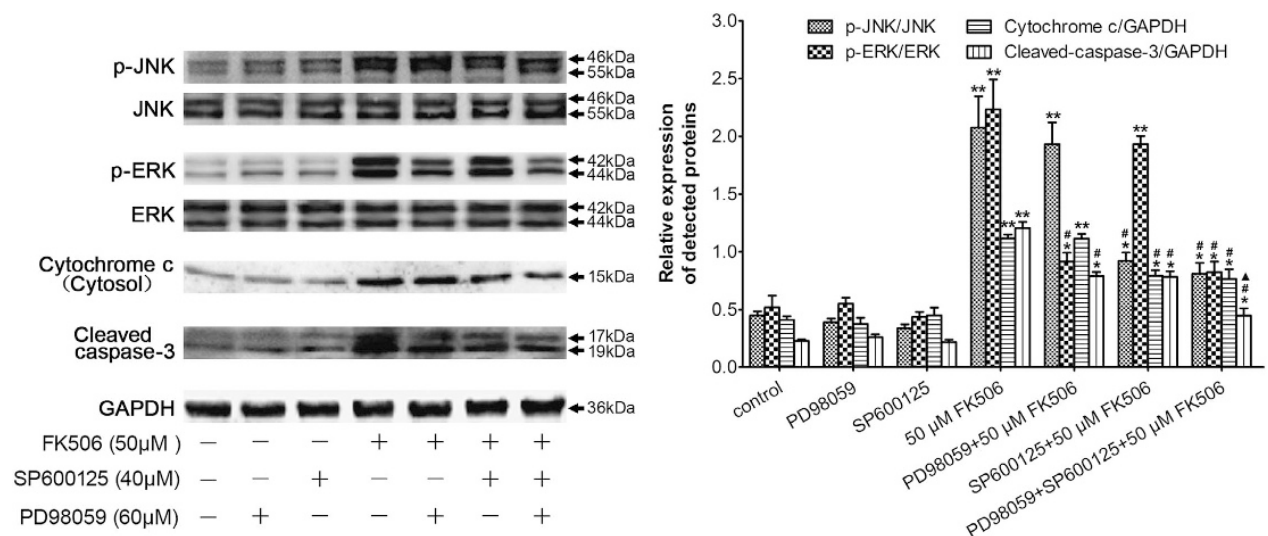

Figure 8 Effect of JNK inhibitor, SP600125, or/and ERK inhibitor, PD98059, on protein expressions of p-JNK, p-ERK, cytosolic cytochrome $c$ and cleaved caspase-3. Cells were pretreated with JNK inhibitor, SP600125 $(40 \mu \mathrm{M})$, or/and ERK inhibitor, PD98059 $(60 \mu \mathrm{M})$, for 30 min before treatment with $50 \mu \mathrm{M}$ FK506 for $8 \mathrm{~h}$, and protein expressions of p-JNK, p-ERK, cytosolic cytochrome $c$ and cleaved caspase-3 were determined using western blotting. JNK inhibitor, SP600125, prevented both phosphorylation of JNK and expressions of cytosolic cytochrome $c$ and cleaved caspase-3. However, ERK inhibitor, PD98059, only prevented the phosphorylation of ERK and the expression of cleaved caspase-3, and had no effect on the expression of cytosolic cytochrome $c$. Moreover, simultaneous application of both inhibitors further decreased the expression of cleaved caspase-3, and almost had no influence on the expressions of p-JNK, p-ERK and cytosolic cytochrome $c$, compared with SP600125 or PD98059 pretreatment, respectively. ${ }^{*} P<0.05,{ }^{* *} P<0.01$ compared with control. ${ }^{\#} P<0.05$ compared with cells treated with $50 \mu \mathrm{M}$ FK506 alone. ${ }^{\boldsymbol{}} P<0.05$ compared with SP600125 or PD98059 pretreatment, respectively

JNK inhibitor, SP600125, or/and by ERK inhibitor, PD98059. JNK and ERK are members of the mitogen-activated protein kinase (MAPK) family. JNK can be rapidly activated by various stress stimuli and mostly mediates apoptosis, ${ }^{22,23}$ whereas the ERK cascade is activated by mitogenic stimuli, and is critical for proliferation and survival. ${ }^{24}$ However, JNK and ERK are controversial in their role in cell death or proliferation. JNK has been reported to induce pro-survival responses in several types of cells ${ }^{25-27}$ and ERK also has been suggested to mediate apoptosis. ${ }^{28-31}$ What determines whether JNK and ERK will act in a pro-apoptotic or pro-survival fashion remains an important unanswered question, but the different stimuli as 
well as the cell type may be determining factors. The role of MAPKs on apoptotic pathways may be unique to the specific model tested, and to the apoptotic trigger assessed. ${ }^{32}$ This study found that FK506 induced sustained activation (phosphorylation) of JNK and ERK in fibroblasts in a dosedependent manner. JNK mostly mediates apoptosis, whereas the accumulation of $\mathrm{p}$-ERK in the cytoplasm raises the possibility that kinase may not be available to act on potential downstream pro-survival targets and may contribute to apoptosis through the activation of cytoplasmic cell death mediators. $^{33}$ SP600125 or PD98059 effectively blocked FK506-induced JNK or ERK activation and attenuated FK506-induced apoptosis, suggesting that the sustained activation of JNK and ERK signaling pathways may be involved in FK506-induced fibroblast apoptosis.

Western blot analysis of cytosolic cytochrome $c$ protein was performed to examine whether FK506 induced fibroblast apoptosis involving the mitochondria. Mitochondria act as an important apparatus for signals during apoptosis, and the loss of mitochondrial membrane integrity can induce the release of cytochrome $c$ from mitochondria into cytosol. ${ }^{34}$ The release of cytochrome $c$ is important in this apoptotic pathway, as cytochrome $c$ binds to apoptotic proteaseactivating factor-1 (Apaf-1) forming a complex that in turn cleaves caspase-3. ${ }^{35}$ In this study, the level of cytosolic cytochrome $c$ significantly increased in a dose-dependent manner after FK506 treatment. After preincubation with JNK inhibitor SP600125, the p-JNK and cytosolic cytochrome $c$ were markedly decreased. Preincubation with ERK inhibitor PD98059, however, resulted in a significant decrease of $p$-ERK, leaving the level of cytosolic cytochrome $c$ almost unchanged. These results indicated that mitochondria have a critical role and that p-JNK, but not p-ERK, is involved in mitochondrial pathway of FK506-induced fibroblast apoptosis.

Caspase- 3 is one of the key agents of apoptosis, as it is either partially or totally responsible for the proteolytic cleavage of many key proteins. ${ }^{36}$ Although $p$-ERK had no effect on the release of cytochrome $c$, it still contributed to the activation of caspase-3. Preincubation with SP600125 or PD98059 both led to a significant decrease of cleaved caspase-3. Moreover, simultaneous application of the two inhibitors had additive roles that further decreased the expression of cleaved caspase-3 and the apoptotic percentage, and almost had no influence on the expressions of p-JNK, p-ERK and cytosolic cytochrome $c$, compared with SP600125 or PD98059 pretreatment, respectively. These observations further demonstrated that JNK and ERK pathways are independently involved, but have additive roles in FK506-induced fibroblast apoptosis.

In conclusion, FK506 activates both JNK and ERK with or without mitochondrial cytochrome $c$ release, followed by the cleavage of caspase-3, subsequently leading to the apoptosis of fibroblasts, and FK506 has a valid effect on reducing scar formation in sciatic nerve-injured rat by inducing fibroblast apoptosis. Further research is needed to study at the effect of FK506 on other extracellular matrix components in the process of scar formation. Experiments will be continuously performed in vitro to further elucidate the signaling pathways of FK506-induced apoptosis in fibroblasts.

\section{Materials and methods}

Animals and establishment of rat sciatic nerve transection anastomosis models. Healthy male Sprague-Dawley rats, weighing 250-300 g were purchased from Shanghai Laboratory Animal Center, the Chinese Academy of Sciences (Shanghai, China). All procedures were approved by the Animal Ethics Committee of Nanjing Medical University in China and were in compliance with the Guide for the Care and Use of Laboratory Animals published by the Chinese National Institute of Health. Rats were anesthetized by $30 \mathrm{mg} / \mathrm{kg}$ sodium pentobarbital via intraperitoneal injection and fixed in an abduction position at room temperature. After the right thigh and buttock were shaved, a longitudinal incision was made along the median femur to expose $\sim 2 \mathrm{~cm}$ of the sciatic nerve. The greater sciatic foramen was cut at the tendon level of the obturator internus muscle. The incision was sutured with three sutures under $\times 10$ magnification using a surgical microscope (Latric Optical Instrument Factory, Shanghai, China) and 10/0 nylon monofilament (Latric Suture Needle Company Ltd., Ningbo, China). Rats were postoperatively injected with penicillin $1600000 \mathrm{U} / \mathrm{kg}$ at the non-operated side to prevent infection.

Drug intervention in vivo. FK506 was purchased from Sigma (St. Louis, MO, USA), containing $1 \mathrm{mg}$ per capsule. After the capsule dressing was removed, the powder was dissolved in saline and prepared into a homogeneous suspension with a final concentration of $0.5 \mathrm{mg} / \mathrm{ml}$ by microwaving for $5 \mathrm{~min}$. Rats were intragastrically injected with the suspension at a dose of $4 \mathrm{mg} / \mathrm{kg}$ per day after model establishment. Thirty rats were randomly divided into three groups (ten rats in each group): model group, saline gavage for 4 weeks after sciatic nerve injury; FK506 group, FK506 gavage for 4 weeks after sciatic nerve injury; normal control group, saline gavage for 4 weeks without sciatic nerve injury.

Harvesting the sciatic nerve. At 4 weeks after model establishment, all the rats of each group were sacrificed. The operative sciatic nerve was completely dissected, a 5-mm-long nerve trunk was cut from $2.5 \mathrm{~mm}$ lateral to the distal and proximal ends of the anastomosis, followed by labeling, conventional fixation, dehydration, transparency, permeation, embedding, sectioning, coating, paraffin mounting and slicing into $4-\mu \mathrm{m}$ thick transversal sections. Twelve successive transversal sections of each rat were made through the anastomosis from the proximal to the distal end.

Masson staining. Six odd sections of each rat were stained with Masson's trichrome. Sections were dewaxed and mordanted (10\% potassium dichromate $+10 \%$ trichloroacetic acid) for $30 \mathrm{~min}$, nuclei were stained with hematoxylin for $20 \mathrm{~min}$, differentiated with hydrochloric acid and ethanol for $15 \mathrm{~s}$, returned to blue with weak ammonia for $15 \mathrm{~s}$, stained with Masson solution (Cell Signaling Technology, Irvine, CA, USA) for 1 min, rinsed with $1 \%$ acetic acid, dehydrated with an increasing ethanol series, cleared with xylene I and II for $10 \mathrm{~min}$ to make sections transparent and finally sealed in resin. Then collagen fiber proliferation was observed under the light microscope (Olympus, Tokyo, Japan) with the magnification of $\times 400$.

Immunohistochemical analysis. Six even sections of each rat were dewaxed and rehydrated through gradient ethanol solutions. The sections were incubated in citrate buffer to activate the antigenicity and exposed to $3 \% \mathrm{H}_{2} \mathrm{O}_{2}$ for $10 \mathrm{~min}$ to block endogenous peroxidase. All subsequent incubations were performed in a humidified chamber at $37^{\circ} \mathrm{C}$ unless otherwise stated. The sections were blocked in common goat serum solution for $15 \mathrm{~min}$, followed by incubation overnight with rabbit polyclonal anti-TGF- $\beta(1: 50)$ at $4{ }^{\circ} \mathrm{C}$. Sections were then washed in phosphate buffered saline (PBS) and incubated with horseradish peroxidase-conjugated goat anti-rabbit lgG antibody for $15 \mathrm{~min}$. The cells were visualized by using $3,3^{\prime}$-diaminobenzidine for $5 \mathrm{~min}$ at room temperature, and the sections were counterstained with hematoxylin for $2 \mathrm{~min}$. The sections were analyzed under light microscope with the magnification of $\times 400$.

Histological image analysis. Six sections of each rat stained with Masson's trichrome were selected and the histological images at $\times 12.5$ magnification were obtained. The areas of scar tissue were automatically calculated by Image-pro plus 5.0 image analysis software (Media Cybernetics, Shanghai, China). The area of scar tissue for each rat was defined as the mean scar area of six sections and expressed in $\mathrm{mm}^{2}$. Another six sections stained with TGF- $\beta$ antibody of each rat were selected, and the histological images at $\times 400$ magnification were also obtained. The counting area was selected and each was about $100 \mu \mathrm{m} \times 100 \mu \mathrm{m}$ from three fields on each section. The number of 
fibroblast was calculated, and the number of fibroblast for each section was defined as the mean number of three fields, and the number for each rat was defined as the mean number of six sections.

Culture of rat skin fibroblasts and drug intervention in vitro. Primary cultures of rat skin fibroblasts were obtained from male Sprague-Dawley newborn rats (Shanghai Laboratory Animal Center, the Chinese Academy of Sciences) 1-2 days after birth as reported previously, ${ }^{37}$ with minor modifications. In brief, after thiopental anesthesia, the skin of dorsal part of the lumbar region was shaved and disinfected with $70 \%$ ethanol. Skin samples were aseptically removed from the rats and then minced and incubated for $3 \mathrm{~h}$ in $0.1 \%$ collagenase solution $\left(37^{\circ} \mathrm{C}, 5 \% \mathrm{CO}_{2}\right)$. After incubation, the cell suspension was centrifuged at $250 \times g$ for $5 \mathrm{~min}$. The supernatant was discarded, and the isolated cells were cultured in DMEM (HyClone, Logan, UT, USA) supplemented with $10 \%$ fetal bovine serum (JRH Biosciences, Lenexa, KS, USA), $100 \mathrm{U} / \mathrm{ml}$ penicillin $\mathrm{G}$ and $100 \mathrm{U} / \mathrm{ml}$ streptomycin. After $2 \mathrm{~h}$, the nonadherent cells were washed out. Cells were cultured in an atmosphere of $5 \% \mathrm{CO}_{2}$ at $37^{\circ} \mathrm{C}$. After confluence was attained, cells were subcultivated by a 5 -min incubation in $0.1 \%$ trypsin and $5 \mathrm{mM}$ ethylenediaminetetraacetic acid (EDTA). The free cells were added to an equal volume of culture medium. Following centrifugation $(1000 \times \mathrm{g}$ for $5 \mathrm{~min})$, cells were resuspended in culture medium. The thin and triangular cells with light cytoplasm present in the culture were identified as fibroblasts. The purity of the cultures and absence of other cells were confirmed by morphological examinations. Supplied as a crystalline solid containing $5 \mathrm{mg}$ per bottle, FK506 was dissolved in DMSO and stored at $-20^{\circ} \mathrm{C}$. The serum-free medium containing different concentrations of FK506 $(12.5 \mu \mathrm{M}, 25,50,75$ and $100 \mu \mathrm{M})$ were freshly prepared for each experiment. Preincubation with JNK inhibitor, SP600125 $(40 \mu \mathrm{M})$, or/and ERK inhibitor, PD98059 $(60 \mu \mathrm{M})$, was carried out for 30 min before cells were treated with $50 \mu \mathrm{M}$ FK506. FK506, DMSO, SP600125 and PD98059 were purchased from Sigma.

CCK-8 assay. Cells were plated in 96-well plates $\left(100 \mu\right.$ l, $5 \times 10^{3}$ per well) and allowed to adhere for $24 \mathrm{~h}$ at $37^{\circ} \mathrm{C}$. The cells were then treated with increasing concentrations of FK506 for $6 \mathrm{~h}$. Following treatments, CCK-8 (Dojindo Laboratories, Kumamoto, Japan) solution $(10 \mu l)$ was added to each well. After incubation at $37^{\circ} \mathrm{C}$ for another $2 \mathrm{~h}$, viable cells were detected by measuring absorbance at $570 \mathrm{~nm}$ using an absorbance microplate reader (ELx800 Absorbance Microplate Reader, Bio-Tek, Seattle, WA, USA). Cell viability was expressed as percentage absorbance of cells treated with FK506 compared with the percentage absorbance of untreated cells.

Flow cytometry. Fibroblasts were plated in 6 -well plates $\left(2 \mathrm{ml}, 1 \times 10^{6}\right.$ per well) and incubated overnight at $37^{\circ} \mathrm{C}$. After FK506 treatment, cells were harvested, washed twice with pre-chilled PBS, resuspended in $1 \times$ binding buffer at $1 \times 10^{6} \mathrm{cell} / \mathrm{s} / \mathrm{ml}$, and then incubated with Annexin V-FITC and Propidium lodide (Invitrogen, Carlsbad, CA, USA) according to the manufacturer's instructions. The mixed solution was gently vortexed and incubated in the dark on ice for $15 \mathrm{~min}$. The apoptosis rate of fibroblasts was detected by an FACSAria flow cytometer with FACSDiva Software (Becton Dickinson, San Jose, CA, USA) within $1 \mathrm{~h}$

Hoechst 33342 staining. Fibroblasts were plated in 6-well plates $(2 \mathrm{ml}$, $1 \times 10^{6}$ per well) and incubated to adhere overnight at $37^{\circ} \mathrm{C}$. Increasing concentrations of FK506 were then added to each well and incubated for another $8 \mathrm{~h}$ at $37^{\circ} \mathrm{C}$. The fibroblastic monolayer was then washed three times with PBS and stained with $5 \mu \mathrm{g} / \mathrm{ml}$ Hoechst 33342 for $30 \mathrm{~min}$ at $37^{\circ} \mathrm{C}$ in the dark. Following staining, fibroblasts were washed three times with PBS. The morphological features of apoptosis were observed by fluorescence microscopy (Olympus BX 51) with the magnification $\times 400$.

Western blotting. Cells were harvested and washed three times with cold PBS. Then cells were lysed with lysis buffer ( $1 \%$ Nonidet P- $40,50 \mathrm{mM}$ Tris, $\mathrm{pH}$ $7.5,100 \mathrm{mM} \mathrm{NaCl}, 5 \mathrm{mM}$ EDTA, $10 \mu \mathrm{g} / \mathrm{ml}$ aprotinin, $10 \mu \mathrm{g} / \mathrm{ml}$ leupeptin and $1 \mathrm{mM}$ PMSF). Lysates was centrifuged at $12000 \times g$ for $30 \mathrm{~min}$ at $4^{\circ} \mathrm{C}$. The supernatant was collected and protein concentrations were determined using BCA assay kit (Pierce, Rockford, IL, USA) according to the manufacturer's instructions. Cellular extract containing $50 \mu \mathrm{g}$ of total protein from each sample were separated by $12 \%$ SDS-PAGE and transferred to PVDF membranes using a standard protocol. The membranes were blocked with TBS- $0.05 \%$ Tween- 20 containing $5 \%(\mathrm{v} / \mathrm{v})$ bovine serum albumin (Sigma) for $90 \mathrm{~min}$ at $37^{\circ} \mathrm{C}$, then incubated with primary antibodies overnight at $4{ }^{\circ} \mathrm{C}$. The following antibodies were used: GAPDH, JNK, ERK, p-JNK, p-ERK (Cell Signaling Technology, 1:1000 dilution) and cleaved caspase-3 (Abcam, Cambridge, UK, 1:200 dilution). The second day, membranes were washed three times and hybridized with horseradish peroxidase-conjugated secondary antibody (Sigma, 1:10000 dilution) for $2 \mathrm{~h}$ at $37^{\circ} \mathrm{C}$. After washing three times, protein bands specific for antibody were visualized by fluorography using an enhanced chemiluminescence kit (Amersham Pharmacia Biotech, Piscataway, NJ, USA). Band densities were quantified using Image-J, a public domain Java image-processing program inspired by NIH and were normalized to GAPDH to correct for variations in protein loading.

Measurement of cytosolic cytochrome $\boldsymbol{c}$. Cells were resuspended in buffer (250 mM sucrose, $20 \mathrm{mM}$ HEPES, $10 \mathrm{mM} \mathrm{KCl}, 1.5 \mathrm{mM} \mathrm{MgCl}$, 1 mM EDTA, $1 \mathrm{mM}$ EGTA, $1 \mathrm{mM} \mathrm{DTT}, 17 \mu \mathrm{g} / \mathrm{ml}$ of PMSF, $8 \mu \mathrm{g} / \mathrm{ml}$ of aprotinin and $2 \mu \mathrm{g} / \mathrm{ml}$ of leupeptin, $\mathrm{pH}$ 7.4) on ice for $30 \mathrm{~min}$ and then homogenized gently with a glass Dounce homogenizer for 20 strokes. The homogenates were centrifuged at $750 \times \mathrm{g}$ for $10 \mathrm{~min}$ at $4{ }^{\circ} \mathrm{C}$ to remove nuclei, and the supernatants were centrifuged at $20000 \times g$ for $30 \mathrm{~min}$ at $4{ }^{\circ} \mathrm{C}$ to remove mitochondria and other insoluble fragments. Protein $(50 \mu \mathrm{g})$ was subjected to $12 \%$ SDS-PAGE, and then western blot analysis was performed using anticytochrome $c$ antibody (Abcam, $1: 500$ dilution).

Statistical analysis. Data are presented as mean \pm S.D. Statistical significance was determined using analysis of variance (ANOVA). A $P$-value of $<0.05$ was considered statistically significant. The correlation between scar area and fibroblast number was analyzed using linear correlation analysis, with $P<0.05$ indicating correlation.

\section{Conflict of Interest}

The authors declare no conflict of interest.

Acknowledgements. This study was supported by grants from the National Natural Science Foundation of China (No. 30973058, 81171694) and the Program for Development of Innovative Research Team in the First Affiliated Hospital of Nanjing Medical University (No. IRT-015). All authors participated in the study design, conduction, evaluation and all contributed to preparation of the manuscript. Jun Que and Quan Cao drafted the manuscript.

1. Sosa I, Reyes O, Kuffler DP. Immunosuppressants: neuroprotection and promoting neurological recovery following peripheral nerve and spinal cord lesions. Exp Neurol 2005; 195: 7-15.

2. Rustemeyer J, Dicke U. Allografting combined with systemic FK506 produces greater functional recovery than conduit implantation in a rat model of sciatic nerve injury. J Reconstr Microsurg 2010; 26: 123-129.

3. Graham WP, Pataky PE, Calabretta AM, Munger BL, Buda MJ. Enhancement of peripheral nerve regeneration with triamcinolone after neurorrhaphy. Surg Forum 1973; 24: 457-459.

4. Lane JM, Bora FW, Pleasure D. Neuroma scar formation in rats following peripheral nerve transection. J Bone Joint Surg Am 1978; 60: 197-203.

5. Sunderland S. Nerve and Nerve Injury. Churchill Livingstone: New York, 1987. pp 173-176.

6. Atkins S, Smith KG, Loescher AR, Boissonade FM, O'Kane S, Ferguson MW et al. Scarring impedes regeneration at sites of peripheral nerve repair. Neuroreport 2006; 17 : 1245-1249

7. Bora FJ. Peripheral nerve repair in cats. The fascicular stitch. J Bone Joint Surg 1967; 49: 659-666

8. Yan Y, Sun HH, Hunter DA, Mackinnon SE, Johnson PJ. Efficacy of short-term FK506 administration on accelerating nerve regeneration. Neurorehabil Neural Repair 2012; 26: 570-580.

9. Jifeng H, Dezhong L, Qiongjiao Y, Huayong Z, Shipu L. Evaluation of PRGD/FK506/NGF conduits for peripheral nerve regeneration in rats. Neurol India 2010; 58: 384-391.

10. Li X, Wang W, Wei G, Wang G, Zhang W, Ma X. Immunophilin FK506 loaded in chitosan guide promotes peripheral nerve regeneration. Biotechnol Lett 2010; 32: 1333-1337.

11. Jost SC, Doolabh VB, Mackinnon SE, Lee M, Hunter D. Acceleration of peripheral nerve regeneration following FK506 administration. Restor Neurol Neurosci 2000; 17: 39-44.

12. Chunasuwankul R, Ayrout C, Dereli Z, Gal A, Lanzetta M, Owen E. Low dose discontinued FK506 treatment enhances peripheral nerve regeneration. Int Surg 2002; 87: 274-278.

13. Becker DB, Jensen JN, Myckatyn TM, Doolabh VB, Hunter DA, Mackinnon SE. Effects of FKBP-12 ligands following tibial nerve injury in rats. J Reconstr Microsurg 2000; 16: $613-620$. 
14. Haisheng $\mathrm{H}$, Songjie $\mathrm{Z}$, Xin $\mathrm{L}$. Assessment of nerve regeneration across nerve allografts treated with tacrolimus. Artif Cells Blood Substit Immobil Biotechnol 2008; 36: 465-474.

15. Chabas JF, Alluin O, Rao G, Garcia S, Lavaut MN, Legre R et al. FK506 induces changes in muscle properties and promotes metabosensitive nerve fiber regeneration. J Neurotrauma 2009; 26: 97-108.

16. Chen $\mathrm{B}$, Song $\mathrm{Y}$, Liu Z. Promotion of nerve regeneration in peripheral nerve by shortcourse FK506 after end-to-side neurorrhaphy. J Surg Res 2009; 152: 303-310.

17. Lagoda G, Xie Y, Sezen SF, Hurt KJ, Liu L, Musicki B et al. FK506 neuroprotection after cavernous nerve injury is mediated by thioredoxin and glutathione redox systems. J Sex Med 2011; 8: 3325-3334.

18. Li Q, Shen TG, Wu YM, Li J, Wang G. Experimental study of electrophysiologic effects of regenerative nerve fibres affected by control releasing FK506. China J Orthop Traumatol 2010; 23: 841-844

19. Que J, Cao Q, Sui T, Du SH, Zhang AL, Kong DC et al. Tacrolimus reduces scar formation and promotes sciatic nerve regeneration. Neural Regen Res 2012; 7: 2500-2506.

20. Kuo YR, Wu WS, Jeng SF, Huang HC, Yang KD, Sacks JM et al. Activation of ERK and p38 kinase mediated keloid fibroblast apoptosis after flashlamp pulsed-dye laser treatment. Lasers Surg Med 2005; 36: 31-37.

21. Sun $Y$, Wang L, Sun S, Liu B, Wu N, Cao. X. The effect of 10 -hydroxycamptothecine in preventing fibroblast proliferation and epidural scar adhesion after laminectomy in rats. Eur J Pharmacol 2008; 593: 44-48.

22. Davis RJ. Signal transduction by the JNK group of MAP kinases. Cell 2000; 103: 239-252.

23. Jeon SH, Park HM, Kim SJ, Lee MY, Kim GB, Rahman MM et al. Taurine reduces FK506induced generation of ROS and activation of JNK and Bax in Madin Darby canine kidney cells. Hum Exp Toxicol 2010; 29: 627-633.

24. Johnson GL, Lapadat R. Mitogen-activated protein kinase pathways mediated by ERK, JNK, and p38 protein kinases. Science 2002; 298: 1911-1912.

25. Dougherty CJ, Kubasiak LA, Prentice H, Andreka P, Bishopric NH, Webster KA. Activation of c-Jun $\mathrm{N}$-terminal kinase promotes survival of cardiac myocytes after oxidative stress. Biochem J 2002; 362: 561-571.

26. Engelbrecht AM, Niesler C, Page C, Lochner A. p38 and JNK have distinct regulatory functions on the development of apoptosis during simulated ischaemia and reperfusion in neonatal cardiomyocytes. Basic Res Cardiol 2004; 99: 338-350.

27. El-Najjar N, Chatila M, Moukadem H, Vuorela H, Ocker M, Gandesiri M et al. Reactive oxygen species mediate thymoquinone-induced apoptosis and activate ERK and JNK signaling. Apoptosis 2010; 15: 183-195.
28. Park C, Jin CY, Kim GY, Choi IW, Kwon TK, Choi BT et al. Induction of apoptosis by esculetin in human leukemia U937 cells through activation of JNK and ERK. Toxicol Appl Pharmacol 2008; 227: 219-228.

29. Yang SH, Wu ZZ, Chien CM, Lo YH, Wu MJ, Chang LS et al. JNK and ERK mitogenactivated protein kinases mediate THDA-induced apoptosis in K562 cells. Cell Biol Toxicol 2008; 24: 291-302.

30. Lin KL, Su JC, Chien CM, Tseng CH, Chen YL, Chang LS et al. Naphtho [1,2-b]furan-4,5dione induces apoptosis and S-phase arrest of MDA-MB-231 cells through JNK and ERK signaling activation. Toxicol In Vitro 2010; 24: 61-70.

31. Eguchi R, Fujimori Y, Takeda H, Tabata C, Ohta T, Kuribayashi K et al. Arsenic trioxide induces apoptosis through JNK and ERK in human mesothelioma cells. J Cell Physiol 2011; 226: 762-768.

32. van den Brink MR, Kapeller R, Pratt JC, Chang JH, Burakoff SJ. The extracellular signalregulated kinase pathway is required for activation-induced cell death of $\mathrm{T}$ cells. J Biol Chem 1999; 274: 11178-11185.

33. Szydlowska K, Gozdz A, Dabrowski M, Zawadzka M, Kaminska B. Prolonged activation of ERK triggers glutamate-induced apoptosis of astrocytes: neuroprotective effect of FK506. J Neurochem 2010; 113: 904-918.

34. Cai J, Yang J, Jones DP. Mitochondrial control of apoptosis: the role of cytochrome c. Biochim Biophys Acta 1998; 1366: 139-149.

35. Zou H, Henzel WJ, Liu X, Lutschg A, Wang X. Apaf-1, a human protein homologous to C. elegans CED-4, participates in cytochrome c-dependent activation of caspase-3. Cell 1997; 90: 405-413.

36. Park JW, Choi YJ, Suh SI, Baek WK, Suh MH, Jin IN et al. Bcl-2 overexpression attenuates resveratrol-induced apoptosis in U937 cells by inhibition of caspase-3 activity. Carcinogenesis 2001; 22: 1633-1639.

37. Blasinska A, Drobnik J. Effects of nonwoven mats of Di-O-butyrylchitin and related polymers on the process of wound healing. Biomacromolecules 2008; 9: 776-782.

(i) (2) (2) Cell Death and Disease is an open-access journal published by Nature Publishing Group. This work is licensed under the Creative Commons Attribution-NonCommercialShare Alike 3.0 Unported License. To view a copy of this license, visit http://creativecommons.org/licenses/by-nc-sa/3.0/ 